\title{
SOLVABILITY AND NUMERICAL SOLUTION OF VARIATIONAL DATA ASSIMILATION PROBLEMS
}

\author{
Victor Shutyaev* \\ Institute of Numerical Mathematics \\ Russian Academy of Sciences, Russia \\ shutyaev@inm.ras.ru
}

\begin{abstract}
A class of quasilinear variational data assimilation problems on the identification of the the initial-value functions is considered for the models governed by evolution equations. The optimality system is reduced to the equation for the control function. The properties of the control equation are studied and the solvability theorems are proved for linear and quasilinear data assimilation optimality systems. The iterative algorithms for solving the problem are formulated and justified.
\end{abstract}

Keywords: Optimal control, data assimilation, iterative algorithms

\section{Statement of Data Assimilation Problem}

Let $H$ and $X$ be real separable Hilbert spaces such that $X$ is imbedded into $H$ continuously and densely, $H^{*}, X^{*}$ are the spaces adjoint to $H$, $X$, respectively. We assume that $H \equiv H^{*}, \quad(\cdot, \cdot)_{L_{2}(0, T ; H)}=(\cdot, \cdot)$, $\|\cdot\|=(\cdot, \cdot)^{1 / 2}$. Let us consider also the spaces $Y^{0}=L_{2}(0, T ; H)$, $Y=L_{2}(0, T ; X), Y^{*}=L_{2}\left(0, T ; X^{*}\right)$ of abstract functions $\varphi(t)$ with the values in $H, X, X^{*}$, respectively, and the space

$$
W=\left\{\varphi \in L_{2}(0, T ; X): \frac{d \varphi}{d t} \in L_{2}\left(0, T ; X^{*}\right)\right\}
$$

with the norm

$$
\|\varphi\|_{W}=\left(\left\|\frac{d \varphi}{d t}\right\|_{L_{2}\left(0, T ; X^{*}\right)}^{2}+\|\varphi\|_{L_{2}(0, T ; X)}^{2}\right)^{1 / 2} .
$$


Let $a(t ; \varphi, \psi)$ be a bilinear form defined for any $t \in[0, T], \varphi, \psi \in X$ and satisfied the inequalities:

$$
\begin{gathered}
|a(t ; \varphi, \psi)| \leq c_{1}\|\varphi\|_{X}\|\psi\|_{X}, \quad c_{1}=\text { const }>0 \\
c_{2}\|\varphi\|_{X}^{2} \leq a(t ; \varphi, \varphi), \quad c_{2}=\text { const }>0, \quad \forall t \in[0, T], \quad \forall \varphi, \psi \in X .
\end{gathered}
$$

By $A(t) \in \mathcal{L}\left(Y, Y^{*}\right)$ we denote the operator generated by this form:

$$
(A(t) \varphi, \psi)_{H}=a(t ; \varphi, \psi) \quad \forall \varphi, \psi \in X .
$$

Consider the following quasilinear evolution problem:

$$
\left\{\begin{array}{l}
\frac{d \varphi}{d t}+A(t) \varphi+\tau F(\varphi)=f(t), \quad t \in(0, T) \\
\varphi(0)=u,
\end{array}\right.
$$

where $f \in Y^{*}, u \in H, \tau \in\left[-\tau_{0}, \tau_{0}\right]$ is a parameter, $\tau_{0} \in \mathbf{R}^{+}, F(\varphi)$ is a nonlinear Frechet differentiable operator, $F: Y \rightarrow Y^{*}$. Introduce a functional of $u \in H$ of the form:

$$
S(u)=\frac{\alpha}{2}\left\|u-\widehat{\varphi}^{0}\right\|_{H}^{2}+\frac{1}{2}\|B \varphi-\widehat{\varphi}\|_{Z}^{2},
$$

where $\alpha=$ const $\geq 0, Z$ is a Hilbert space (observational space) with the scalar product $(\cdot, \cdot)_{Z}$ and the norm $\|\cdot\|_{Z}=(\cdot, \cdot)_{Z}^{1 / 2}, B: Y \rightarrow Z$ is a linear bounded operator, $\widehat{\varphi}^{0} \in H, \widehat{\varphi} \in Z$. The functions $\widehat{\varphi}^{0}, \widehat{\varphi}$ are generally determined by a priory observational data. The coefficient $\alpha$ is a regularization parameter [1].

Consider the following data assimilation problem: for given $f \in Y^{*}$, $\widehat{\varphi} \in Z$, find $u \in H, \varphi \in W$ such that

$$
\left\{\begin{aligned}
\frac{d \varphi}{d t}+A(t) \varphi+\tau F(\varphi) & =f, t \in(0, T) \\
\varphi(0) & =u \\
S(u) & =\min _{\tilde{u} \in H} S(\tilde{u}) .
\end{aligned}\right.
$$

The problems of the form (1.6) were studied by L.S.Pontryagin [7], J.L.Lions [5], [2] and many others (see Refs.)

The necessary optimality condition [5]reduces the problem (1.6) to the system for finding the functions $\varphi, \varphi^{*} \in W, u \in H$, of the form:

$$
\begin{aligned}
\frac{\varphi}{d t}+A(t) \varphi+\tau F(\varphi) & =f, t \in(0, T) ; \quad \varphi(0)=u \\
-\frac{d \varphi^{*}}{d t}+A^{*}(t) \varphi^{*}+\tau\left(F^{\prime}(\varphi)\right)^{*} \varphi^{*} & =C \widehat{\varphi}-K \varphi, t \in(0, T) ; \quad \varphi^{*}(T)=0,
\end{aligned}
$$




$$
\alpha\left(u-\widehat{\varphi}^{0}\right)-\varphi^{*}(0)=0,
$$

where $\left(F^{\prime}(\varphi)\right)^{*}: Y \rightarrow Y^{*}$ is the operator adjoint to the Frechet derivative of $F$ at the point $\varphi \in W, A^{*}(t): Y \rightarrow Y^{*}$ is adjoint to $A(t), K: Y \rightarrow$ $Y^{*}, C: Z \rightarrow Y^{*}$ are linear bounded operators, $K=C B, C$ is defined by the equality $(C \theta, \psi)=(\theta, B \psi)_{Z} \forall \theta \in Z, \psi \in Y$, and equations (1.7), (1.8) are considered in the space $Y^{*}$.

\section{Linear Data Assimilation Problem}

Consider the problem (1.7)-(1.9) for $\tau=0$. The solutions of problems (1.7), (1.8) for $\tau=0$ may by represented [6]as

$$
\varphi=G_{0} u+G_{1} f, \varphi^{*}=G_{1}^{(T)}(C \widehat{\varphi}-K \varphi),
$$

where $G_{0}: H \rightarrow W, G_{1}: Y^{*} \rightarrow W, G_{1}^{(T)}: Y^{*} \rightarrow W$ are linear bounded operators. Eliminating $\varphi, \varphi^{*}$ from (1.7)-(1.9) for $\tau=0$, we come to the equation for the control $u$ :

$$
L u=P,
$$

where the operator $L: H \rightarrow H$ and the right-hand side $P$ are defined by

$$
L=\alpha E+T_{0} G_{1}^{(T)} K G_{0}, \quad P=\alpha \widehat{\varphi}^{0}+T_{0} G_{1}^{(T)} C \widehat{\varphi}-T_{0} G_{1}^{(T)} K G_{1} f,
$$

$E$ is the identity operator, $T_{0}: W \rightarrow H$ is the trace operator: $T_{0} \varphi=$ $\left.\varphi\right|_{t=0}$.

Consider the operator $L$ for $\alpha=0$ and denote it by $\bar{L}$. Let $G_{0}: H \rightarrow$ $W$ be the operator from (2.1), where the element $G_{0} u$ is defined as the solution of (1.7) for $\tau=0, f=0$. The following statement holds.

LEMMA 1 The operator $\bar{L}: H \rightarrow H$ is continuous, self-adjoint, and positive semi-definite:

$$
(\vec{L} v, v)_{H} \geq 0 \quad \forall v \in H .
$$

If the operator $B G_{0}: H \rightarrow Z$ is invertible, the operator $\bar{L}$ is positive: $(\bar{L} v, v)_{H}>0 \quad \forall v \in H, v \neq 0$.

Proof. Let $\rho \in H$ and $\varphi=G_{0} \rho$. Then

$$
\bar{L} \rho=T_{0} G_{1}^{(T)} K \varphi \text {. }
$$

The first assertion of Lemma 1 was proved in [12]. The positive definiteness or semi-definiteness of $\bar{L}$ follow from the equalities:

$$
(\bar{L} \rho, \rho)_{H}=\left(T_{0} G_{1}^{(T)} K G_{0} \rho, \rho\right)_{H}=(K \varphi, \varphi)=(B \varphi, B \varphi)_{Z}=\left\|B G_{0} \rho\right\|_{Z}^{2} .
$$


The lemma is proved.

From Lemma 1, we get

LEMma 2 If the operator $B G_{0}: H \rightarrow Z$ is invertible, then the range $R(\bar{L})$ of the operator $\bar{L}$ is dense in $H$, and the equation $\bar{L} u=P$ is solvable uniquely and densely in $H$.

Remark 1. In case of "complete observation", when $Z=Y^{0}, B=E$ (the identity operator), we have $C=E, K=E$, and the operator $\vec{L}$ is positive.

Introduce the following additional restriction on the operator $A(t)$ :

Hypothesis (A): For any $p \in Y^{0}$ the solution $\varphi^{*}$ of the adjoint problem

$$
-\frac{d \varphi^{*}}{d t}+A^{*}(t) \varphi^{*}=p, t \in(0, T) ; \quad \varphi^{*}(T)=0
$$

satisfies the inequality $\left\|\varphi^{*}(0)\right\|_{X} \leq c\|p\|_{Y^{0}}, c=$ const $>0$.

Remark 2. The hypothesis $(\bar{A})$ is satisfied for a wide class of operators $A(t)$, among them - the second-order elliptic operators in uniformly parabolic problems [4], [12].

LEMMA 3 Let $X$ be compactly imbedded into $H$, the hypothesis (A) be satisfied, and the operator $K: Y^{0} \rightarrow Y^{0}$ be bounded. Then the operator $\bar{L}: H \rightarrow H$ is compact.

Proof. Let us prove that $\bar{L}$ maps a bounded set of $H$ into a compact set. Consider $u \in H$ such that $\|u\|_{H} \leq c_{0}, c_{0}=$ const $>0$. Let $\varphi=G_{0} u, \varphi^{*}=G_{1}^{(T)} K \varphi$, then $\bar{L} u=\varphi^{*}(0)$. Since

$$
\|\varphi\|_{W} \leq c_{1}\|u\|_{H},\left\|\varphi^{*}\right\|_{W} \leq c_{2}\|K \varphi\|_{Y^{*}}, \quad c_{1}, c_{2}=\text { const }>0,
$$

and by the hypothesis $(\mathrm{A})$,

$$
\left\|\varphi^{*}(0)\right\|_{X} \leq c\|K \varphi\|_{Y^{0}}, c=\text { const }>0,
$$

then, due to the boundedness of $K: Y^{0} \rightarrow Y^{0}$, we get

$$
\|\bar{L} u\|_{X} \leq c_{3}\|u\|_{H} \leq c_{3} c_{0}
$$

where $c_{3}=$ const $>0$. However, $X$ is compactely imbedded into $H$, hence the set $M=\left\{\bar{L} u:\|u\|_{H} \leq c_{0}\right\}$ is compact in $H$, i.e. the operator $\bar{L}: H \rightarrow H$ is compact.

LEMMA 4 The spectrum $\sigma(\bar{L})$ of the operator $\bar{L}$ satisfies

$$
0 \leq \sigma(\bar{L}) \leq \nu^{2}\|B\|^{2}
$$


with the constant $\nu$ from the inequality $\|\varphi\|_{Y} \leq \nu\|u\|_{H}$, where $u \in H$, and $\varphi=G_{0} u$ is the solution of the problem $\frac{d \varphi}{d t}+A(t) \varphi=0, t \in$ $(0, T) ; \quad \varphi(0)=u$.

Proof. To estimate the spectrum of the self-adjoint operator $\bar{L}$ consider $(\bar{L} u, u)$ for $u \in H$. Let $\varphi=G_{0} u, \varphi^{*}=G_{1}^{(T)} \varphi$, then

$$
\begin{gathered}
(\bar{L} u, u)_{H}=\left(\varphi^{*}(0), u\right)_{H}=(K \varphi, \varphi)=\|B \varphi\|_{Z}^{2} \\
\leq\|B\|^{2}\|\varphi\|_{Y}^{2} \leq \nu^{2}\|B\|^{2}\|u\|_{H}^{2} .
\end{gathered}
$$

Hence,

$$
\sigma(\bar{L}) \leq \sup _{u \in H, u \neq 0} \frac{(\bar{L} u, u)}{(u, u)} \leq \nu^{2}\|B\|^{2} .
$$

This ends the proof.

For case of complete observation, from Lemmas 1-3 we have the following

LEMMA 5 Let $Z=Y^{0}, B=E$ (the identity operator), $X$ be compactly imbedded into $H$ and the hypothesis (A) be satisfied. Then the operator $\bar{L}^{-1}: H \rightarrow H$ exists, being unbounded; zero is the point of the continuous spectrum of the operator $\bar{L}$; the equation $\bar{L} u=P$ is solvable in $H$ if and only if

$$
\sum_{k=1}^{\infty} \mu_{k}^{-2}\left(P, u_{k}\right)_{H}^{2}<\infty,
$$

where $u_{k}$ is the orthonormal system of the eigenfunctions of the compact operator $\bar{L}$, corresponding to the eigenvalues $\mu_{k}$.

The spectrum bounds of the operator $L$ are very important for justification and optimization of iterative algorithms for solving the original data assimilation problem. Some estimates for the spectrum bounds may be derived using Lemma 4 . If $K=E$, for the spectrum $\sigma(L)$ of the operator $L$ defined by (2.2) the following estimates hold [11]:

$$
m \leq \sigma(L) \leq M
$$

where

$$
m=\alpha+\int_{0}^{T} e^{-\int_{0}^{t} \lambda_{\max }(\tau) d \tau} d t, \quad M=\alpha+\int_{0}^{T} e^{-\int_{0}^{t} \lambda_{\min }(\tau) d \tau} d t
$$

and $\lambda_{\min }, \lambda_{\max }$ are the lower and upper bounds, respectively, of the spectrum of the operator $A+A^{*}$. 
If $K=E$, and $A(t)=A: H \rightarrow H$ is a linear closed operator independent of time, being unbounded self-adjoint positive definite operator in $H$ with the compact inverse, then the eigenvalues $\mu_{k}$ of the operator $\bar{L}$ are defined by the formula [11]:

$$
\mu_{k}=\frac{1-e^{-2 \lambda_{k} T}}{2 \lambda_{k}}
$$

where $\lambda_{k}$ are the eigenvalues of the operator $A$. In this case the estimates (2.7) are exact, because in (2.7) $\lambda_{\min }=2 \lambda_{1}, \lambda_{\max }=\infty$, and $m, M$ are given in the explicit form:

$$
m=\alpha, \quad M=\alpha+\frac{1-e^{-2 \lambda_{1} T}}{2 \lambda_{1}} .
$$

where $\lambda_{1}$ is the least eigenvalue of the operator $A$.

From Lemma 1 it follows that for $\alpha>0$ the operator $L: H \rightarrow H$ is positive definite (i.e. coercive). Then, using the well-known results on solvability of linear optimal control problems [5] we come to the solvability theorem for the linear problem (1.7)-(1.9):

Theorem 6 Let $f \in Y^{*}, \widehat{\varphi}^{0} \in H, \widehat{\varphi} \in Z$. Then for $\alpha>0$ the problem (1.7)-(1.9) for $\tau=0$ has a unique solution $\varphi_{0} \in W, \varphi_{0}^{*} \in W, u_{0} \in H$, and the following estimate holds:

$\left\|\varphi_{0}\right\|_{W}+\left\|\varphi_{0}^{*}\right\|_{W}+\left\|u_{0}\right\|_{H} \leq c_{0}\left(\left\|\widehat{\varphi}^{0}\right\|_{H}+\|C \widehat{\varphi}\|_{Y^{*}}+\|f\|_{Y^{*}}\right), c_{0}=$ const $>0$

\section{Solvability of Nonlinear Problem}

Let $c_{0}$ be the constant from (2.9). The following theorem holds:

THEOREM 7 Let $f \in Y^{*}, \widehat{\varphi}^{0} \in H ; \hat{\varphi} \in Z$ and for some $R>0$ the inequalities

$$
\left\|F^{\prime}(\xi)\right\|_{Y \rightarrow Y^{*}} \leq k_{1}, \quad\left\|F^{\prime}(\xi)-F^{\prime}(\eta)\right\|_{Y \rightarrow Y^{*}} \leq k_{2}\|\xi-\eta\|_{W}
$$

are satisfied for any $\xi, \eta \in B\left(\varphi_{0}, R\right)=\left\{\varphi \in Y:\left\|\varphi-\varphi_{0}\right\|_{W} \leq R\right\}$, where $k_{i}=k_{i}\left(\varphi_{0}, R\right)=$ const $>0$. Then for $|\tau| \leq \tau_{0}$, with

$$
\tau_{0}=1 / c_{0}\left[k_{1}+k_{2}\left(R+\left\|\varphi_{0}^{*}\right\|_{W}\right)+\frac{1}{R}\left(\left\|F\left(\varphi_{0}\right)\right\|_{Y^{*}}+k_{1}\left\|\varphi_{0}^{*}\right\|_{W}\right)\right]^{-1},
$$

the problem (1.2)-(1.4) has a unique solution $\left(\varphi, \varphi^{*}, u\right) \in W \times W \times H$.

Proof. Consider the problem for the remainders $\tilde{\varphi}=\varphi-\varphi_{0}, \tilde{\varphi}^{*}=$ $\varphi^{*}-\varphi_{0}^{*}, \tilde{u}=u-u_{0}$, where $\left(\varphi_{0}, \varphi_{0}^{*}, u_{0}\right)$ is the solution to the problem 
(1.7)-(1.9) for $\tau=0$. The problem for $\tilde{\varphi}, \tilde{\varphi}^{*}, \tilde{u}$ reads:

$$
\begin{gathered}
\frac{d \tilde{\varphi}}{d t}+A(t) \tilde{\varphi}+\tau F\left(\varphi_{0}+\tilde{\varphi}\right)=0, t \in(0, T) ; \quad \tilde{\varphi}(0)=\tilde{u} \\
-\frac{d \tilde{\varphi}^{*}}{d t}+A^{*}(t) \tilde{\varphi}^{*}+\tau\left(F^{\prime}\left(\varphi_{0}+\tilde{\varphi}\right)\right)^{*}\left(\varphi_{0}^{*}+\tilde{\varphi}^{*}\right)=-K \tilde{\varphi}, t \in(0, T) \\
\tilde{\varphi}^{*}(T)=0 \\
\alpha \tilde{u}-\tilde{\varphi}^{*}(0)=0 .
\end{gathered}
$$

Consider the following iterative process:

$$
\begin{gathered}
\frac{d \tilde{\varphi}^{(n+1)}}{d t}+A(t) \tilde{\varphi}^{(n+1)}+\tau F\left(\tilde{\varphi}^{(n)}+\varphi_{0}\right)=0, t \in(0, T) \\
\tilde{\varphi}^{(n+1)}(0)=\tilde{u}^{(n+1)}, \\
-\frac{d \tilde{\varphi}^{*(n+1)}}{d t}+A^{*}(t) \tilde{\varphi}^{*(n+1)}+\tau\left(F^{\prime}\left(\tilde{\varphi}^{(n)}+\varphi_{0}\right)\right)^{*}\left(\tilde{\varphi}^{*(n)}+\varphi_{0}^{*}\right)=-K \tilde{\varphi}^{(n+1)}, \\
\tilde{\varphi}^{*(n+1)}(T)=0, \\
\alpha \tilde{u}^{(n+1)}-\tilde{\varphi}^{*(n+1)}(0)=0
\end{gathered}
$$

for $\left\|\tilde{\varphi}^{(0)}\right\|_{W}+\left\|\tilde{\varphi}^{*(0)}\right\|_{W} \leq R$. Since (for a fixed $\left.n\right) \tilde{\varphi}^{(n+1)}, \tilde{\varphi}^{*(n+1)}, \tilde{u}^{(n+1)}$ is the solution of the linear problem, then, in view of (3.1), it is easily seen that

$\left\|\tilde{\varphi}^{(n+1)}\right\|_{W}+\left\|\tilde{\varphi}^{*(n+1)}\right\|_{W}+\left\|\tilde{u}^{(n+1)}\right\|_{H} \leq k|\tau|\left(\left\|\tilde{\varphi}^{(n)}\right\|_{W}+\left\|\tilde{\varphi}^{*(n)}\right\|_{W}\right)+f_{0}$, where

$$
k=c_{0}\left(k_{1}+k_{2}\left(R+\left\|\varphi_{0}^{*}\right\|_{W}\right)\right), \quad f_{0}=c_{0}|\tau|\left(\left\|F\left(\varphi_{0}\right)\right\|_{Y^{*}}+k_{1}\left\|\varphi_{0}^{*}\right\|_{W}\right) .
$$

By successive use of the last inequality, we get

$$
\begin{aligned}
\left\|\tilde{\varphi}^{(n)}\right\|_{W} & +\left\|\tilde{\varphi}^{*(n)}\right\|_{W}+\left\|\tilde{u}^{(n)}\right\|_{H} \leq(k|\tau|)^{n}\left(\left\|\tilde{\varphi}^{(0)}\right\|_{W}+\left\|\tilde{\varphi}^{*(0)}\right\|_{W}\right)+ \\
& +\frac{1-(k|\tau|)^{n}}{1-k|\tau|} f_{0} \leq(k|\tau|)^{n} R+\frac{1-(k|\tau|)^{n}}{1-k|\tau|} f_{0} \leq R
\end{aligned}
$$

if $|\tau| \leq \tau_{0}$. Then, consider the problem for $\tilde{\varphi}^{(n+1)}-\tilde{\varphi}^{(n)}, \tilde{\varphi}^{*(n+1)}-$ $\tilde{\varphi}^{*(n)}, \tilde{\tilde{u}}^{(n+1)}-\tilde{u}^{(n)}$. This leads to the estimate:

$$
\begin{gathered}
\left\|\tilde{\varphi}^{(n+1)}-\tilde{\varphi}^{(n)}\right\|_{W}+\left\|\tilde{\varphi}^{*(n+1)}-\tilde{\varphi}^{*(n)}\right\|_{W}+\left\|\tilde{u}^{(n+1)}-\tilde{u}^{(n)}\right\|_{H} \leq \\
\leq k|\tau|\left(\left\|\tilde{\varphi}^{(n)}-\tilde{\varphi}^{(n-1)}\right\|_{W}+\left\|\tilde{\varphi}^{*(n)}-\tilde{\varphi}^{*(n-1)}\right\|_{W}\right),
\end{gathered}
$$


which implies

$$
\tilde{\varphi}^{(n)} \rightarrow \tilde{\varphi}, \quad \tilde{\varphi}^{*(n)} \rightarrow \tilde{\varphi}^{*}, \quad \tilde{u}^{(n)} \rightarrow \tilde{u} \text { as } n \rightarrow \infty, \text { for }|\tau| \leq \tau_{0},
$$

where $\tilde{\varphi}, \tilde{\varphi}^{*}, \tilde{u}$ is the solution to the problem (3.4)-(3.6), and the convergence rate estimate holds:

$$
\left\|\tilde{\varphi}^{(n)}-\tilde{\varphi}\right\|_{W}+\left\|\tilde{\varphi}^{*(n)}-\tilde{\varphi}^{*}\right\|_{W}+\left\|\tilde{u}^{(n)}-\tilde{u}\right\|_{H} \leq c \frac{(k|\tau|)^{n}}{1-k|\tau|}
$$

with $c=$ const $>0$. It is easily seen that for $|\tau| \leq \tau_{0}$ this solution is unique and satisfies the condition $\|\tilde{\varphi}\|_{W}+\left\|\tilde{\varphi}^{*}\right\|_{W}+\|\tilde{u}\|_{H} \leq R$. Thus, under the hypotheses of Theorem, there exists a unique solution of the problem (1.7)-(1.9). Theorem is proved.

If the operator $F(\varphi)$ is analytic, then the functions $\left(\varphi, \varphi^{*}, u\right)$ are represented as the series in the powers of $\tau$ :

$$
\varphi=\varphi_{0}+\sum_{i=1}^{\infty} \tau^{i} \varphi_{i}, \quad \varphi^{*}=\varphi_{0}^{*}+\sum_{i=1}^{\infty} \tau^{i} \varphi_{i}^{*}, \quad u=u_{0}+\sum_{i=1}^{\infty} \tau^{i} u_{i},
$$

convergent for $|\tau|<\tau_{0}$ in $W, W, H$, respectively, where $\varphi_{i}, \varphi_{i}^{*}, u_{i}$ may be found by the small parameter method [8].

\section{Iterative Algorithms}

To solve (1.7)-(1.9) one may use the successive approximation method (3.7)-(3.9). Each step of this method involves a linear data assimilation problem of the form (1.7)-(1.9) for $\tau=0$. To solve it we consider a class of iterative algorithms:

$$
\begin{gathered}
\frac{d \varphi^{k}}{d t}+A(t) \varphi^{k}=f, t \in(0, T) ; \quad \varphi^{k}(0)=u^{k} \\
-\frac{d \varphi^{* k}}{d t}+A^{*}(t) \varphi^{* k}=C \widehat{\varphi}-K \varphi^{k}, t \in(0, T) ; \quad \varphi^{* k}(T)=0 \\
u^{k+1}=u^{k}-\alpha_{k+1} B_{k}\left(\alpha\left(u^{k}-\widehat{\varphi}^{0}\right)-\left.\varphi^{* k}\right|_{t=0}\right)+\beta_{k+1} C_{k}\left(u^{k}-u^{k-1}\right),
\end{gathered}
$$

where $B_{k}, C_{k}: H \rightarrow H$ are some operators, and $\alpha_{k+1}, \beta_{k+1}$ the iterative parameters.

Let $\gamma=\nu^{2}\|B\|^{2}$ with $\nu$ defined in (2.5). We introduce the following notations:

$$
\begin{gathered}
\tau_{o p t}=2(2 \alpha+\gamma)^{-1}, \quad \theta=(2 \alpha+\gamma) \gamma^{-1}, \\
\tau_{k}=2\left(2 \alpha+\gamma-\gamma \cos \omega_{k} \pi\right)^{-1}, \quad k=1,2, \ldots, s,
\end{gathered}
$$




$$
\begin{aligned}
& \alpha_{k+1}=\left\{\begin{array}{cc}
2(2 \alpha+\gamma)^{-1}, & k=0 \\
4 \gamma^{-1} \frac{T_{k}(\theta)}{T_{k+1}(\theta)}, & k>0
\end{array} ;\right. \\
& \beta_{k+1}=\left\{\begin{aligned}
0, & k=0 \\
\frac{T_{k-1}(\theta)}{T_{k+1}(\theta)}, & k>0
\end{aligned}\right. \\
& e_{k}= \begin{cases}0, & k=0 \\
p_{k}\left\|\xi^{k}\right\|_{H}^{2} /\left\|\xi^{k-1}\right\|_{H}^{2}, & k>0,\end{cases} \\
& p_{k+1}=\alpha+\left(K \eta^{k}, \eta^{k}\right) /\left\|\xi^{k}\right\|_{H}^{2}-e_{k}, \quad k=0,1, \ldots,
\end{aligned}
$$

where $\omega_{k}=(2 i-1) / 2 s, T_{k}$ is the $k$-th degree Chebyshev polynomial of the first kind, $\xi^{k}=\alpha\left(u^{k}-\widehat{\varphi}^{0}\right)-\varphi^{* k}(0)$, and $\eta^{k}$ is the solution of the problem $\frac{d \eta^{k}}{d t}+A \eta^{k}=0, t \in(0, T) ; \quad \eta^{k}(0)=\xi^{k}$.

TheOREM 8 (I) If $\alpha_{k+1}=\tau, B_{k}=E, \beta_{k+1}=0,0<\tau<2 /(\alpha+\gamma)$, then the iterative process (4.1)-(4.3) is convergent. For $\tau=\tau_{\text {opt }}$ defined by (4.4) the following convergence rate estimates are valid:

$$
\left\|\varphi-\varphi^{k}\right\|_{W} \leq c_{1} q_{k}, \quad\left\|\varphi^{*}-\varphi^{* k}\right\|_{W} \leq c_{2} q_{k}, \quad\left\|u-u^{k}\right\|_{H} \leq c_{3} q_{k},
$$

where $q_{k}=1 / \theta^{k}, \theta$ is given by (4.4), and the constants $c_{1}, c_{2}, c_{3}, c_{4}$ do not depend on the number of iterations and on the functions $\varphi, \varphi^{k}, \varphi^{*}, \varphi^{* k}$, $u, u^{k}, k>0$.

(II) If $B_{k}=E, \beta_{k+1}=0$, and $\alpha_{k+1}=\tau_{k}$, where the parameters $\tau_{k}$ are defined by (4.5) and repeated cyclically with the period $s$, then the error in the iterative process (4.1)--(4.3) is suppressed after each cycle of the length $s$. After $k=l s$ iterations the error estimates (4.9) are valid with $q_{k}=\left(T_{s}(\theta)\right)^{-l}$.

(III) If $B_{k}=C_{k}=E$ and $\alpha_{k+1}, \beta_{k+1}$ are defined by (4.6), then the error in the algorithm (4.1)-(4.3) is suppressed for each $k \geq 1$, and the estimates (4.9) hold for $q_{k}=\left(T_{k}(\theta)\right)^{-1}$.

(IV) If $B_{k}=C_{k}=E$ and $\alpha_{k+1}=1 / p_{k+1}, \beta_{k+1}=e_{k} / p_{k+1}$, where $e_{k}, p_{k+1}$ are defined by (4.7), (4.8), then the iterative process (4.1)-(4.3) is convergent, and the convergence rate estimates (4.9) are valid with $q_{k}=\left(T_{k}(\theta)\right)^{-1}$. 


\section{Proof.}

The iterative process (4.1)-(4.3) is equivalent to the following iterative algorithm [12]:

$$
u^{k+1}=u^{k}-\alpha_{k+1} B_{k}\left(L u^{k}-P\right)+\beta_{k+1} C_{k}\left(u^{k}-u^{k-1}\right)
$$

for solving the control equation $L u=P$, where $L$ and $P$ are defined in $(2.2)$.

According to Lemma 4, the bounds of the spectrum of the control operator $L$ are given by

$$
m \stackrel{\text { def }}{=} \inf _{u \in H, u \neq 0} \frac{(L u, u)}{(u, u)} \geq \alpha, M \stackrel{\text { def }}{=} \sup _{u \in H, u \neq 0} \frac{(L u, u)}{(u, u)} \leq \alpha+\nu^{2}\|B\|^{2} .
$$

Thus, for $\alpha>0$ for solving the equation $L u=P$ we may use the well-known iterative algorithms with optimal choice of parameters. The theory of these methods is well developed [9]. Taking into account the explicit form of the bounds for $m$ and $M$ from (4.11) and applying for the equation $L u=P$ the simple iterative method, the Chebyshev acceleration methods ( $s$-cyclic and two-step ones), and the conjugate gradient method in the form (4.10), we arrive at the conclusions of Theorem, using the well-known convergence results [9]for these methods. Theorem is proved.

In case $\alpha_{k}=1 / \alpha, B_{k}=E, \beta_{k}=0$, the iterative algorithm (4.1)-(4.3) coincides with the Krylov-Chernousko method [3].

The numerical analysis of the above-formulated iterative algorithms has been done in [10]for the data assimilation problem with a linear parabolic state equation.

\section{References}

[1] A.N.Tikhonov. On the solution of ill-posed problems and the regularization method. Dokl. Akad. Nauk SSSR, 151:501-504, 1963.

[2] J.-L.Lions. On controllability of distributed system. Proc. Natl. Acad. Sci. USA, 94:4828-4835, 1997.

[3] I.A. Krylov and F.L. Chernousko. On a successive approximation method for solving optimal control problems. Zh. Vychisl. Mat. Mat. Fiz., 2:1132-1139, 1962.

[4] O.A. Ladyzhenskaya, V.A. Solonnikov, and N.N. Uraltseva. Linear and Quasilinear Equations of Parabolic Type. Nauka, Moscow, 1967.

[5] J.L. Lions. Contrôle Optimal des Systèmes Gouvernés par des Équations aux Dérivées Partielles. Dunod, Paris, 1968.

[6] J.L. Lions and E.Magenes. Problémes aux Limites non Homogenes et Applications. Dunod, Paris, 1968. 
[7] L.S.Pontryagin, V.G.Boltyanskii, R.V.Gamkre lid ze, and E.F.Mi schen ko. The Mathematical Theory of Optimal Processes. John Wiley, New York, 1962.

[8] G.I. Marchuk, V.I. Agoshkov, and V.P. Shutyaev. Adjoint Equations and Perturbation Algorithms in Nonlinear Problems. CRC Press Inc., New York, 1996.

[9] G.I. Marchuk and V.I. Lebedev. Numerical Methods in the Theory of Neutron Transport. Harwood Academic Publishers, New York, 1986.

[10] E.I. Parmuzin and V.P. Shutyaev. Numerical analysis of iterative methods for solving evolution data assimilation problems. Russ. J. Numer. Anal. Math. Modelling, 14:265-274, 1999.

[11] V.P. Shutyaev. Some properties of the control operator in a data assimilation problem and algorithms for its solution. Differential Equations, 12:2035-2041, 1995.

[12] V.P.Shutyaev. Control operators and iterative algorithms for variational data assimilation problems. Nauka, Moscow, 2001. 